\title{
IMPACT OF ORBICULARIS OCULI MUSCLE STRIP EXCISION DURING UPPER LID BLEPHAROPLASTY ON TEAR FILM BREAK UP TIME AND POSTOPERATIVE DRY EYE SYMPTOMS
}

\author{
By \\ Mostafa F Mohammed \\ Ophthalmology Department, Faculty of Medicine, Al-Azhar University, Cairo, Egypt \\ E-mail: mostafafarid80@gmail.com; Tel.:00201061014849-00966545636346
}

\begin{abstract}
Background: Dry eye syndrome (DES) is common after upper lid blepharoplasty (ULB), but tends to be self-limiting over the course of days to weeks. This occurs because the blink mechanism and tear film spread over the cornea are disrupted temporarily. More aggressive surgical techniques and over-excision of skin and orbicularis oculi muscle (OOM) appeared to increase the risk of dry eye following ULB.
\end{abstract}

Objectives: to study the impact of OOM strip excision during ULB on the tear film break up time (TFBUT) and postoperative occurrence and severity of dry eye symptoms.

Paitents and Methods: This interventional comparative study included 40 eyes of 20 patients (14 females and 6 males, the mean $(\mathrm{M})$ age was $57.05 \pm 4.80$ years old. All patients had bilateral aesthetic ULB. Patients were divided into two groups: Group A: 8 patients ( 5 females and 3 males, $\mathrm{M}$ age was $58.38 \pm 5.53$ years old) who had bilateral ULB surgeries including OOM strip excision. And Group B: 12 patients (9 females and 3 males, $M$ age was $56.17 \pm 4.26$ years old) who had bilateral ULB surgeries with preservation of OOM.

Preoperative evaluation: General medical history and full ophthalmologic history were taken. Full ophthalmologic examination was performed including corneal and tear film examination, TFBUT and Schirmer's tests. Lid crease height, palpebral fissure and marginal reflex distance were measured. Patients with uncontrolled chronic systemic diseases were excluded. Patients with exophthalmos, lid retraction, ptosis, brow ptosis, entropion or ectropion were also excluded.

Follow up: TFBUT and dry eye symptoms were assessed for each patient at one week and at one month postoperatively. Patients with continuous symptoms of dry eye postoperatively were followed up until three months. The changes of TFBUT of participants were statistically analyzed using the paired t-test. The M and standard deviation (SD) were used to obtain probability (P) value which was considered statistically significant when it was $\leq 0.05$.

Results: As regard changes of TFBUT; there was statistically significant decrease in TFBUT M values in group A from $11 \pm 1.37$ seconds (s) preoperatively to be $9.69 \pm 2.18 \mathrm{~s}$ at one week postoperatively. This decrease in TFBUT M value was temporary and reversible as there was statistically significant increase of TFBUT M value from $9.69 \pm 2.18 \mathrm{~s}$ at one week postoperatively to be $10.56 \pm 1.86 \mathrm{~s}$ at one month postoperatively which was near to the preoperative value As regard group B, there were non-statistically significant changes in TFBUT M values. Dry eye symptoms had been recorded by four patients from group A (50\%) and two patients from group B (16.7\%). These symptoms were more severe in patients of group A. 
All these symptoms were reversible and frequent use of lubricating eye drops and gels for one week was enough to reduce the patients' discomfort in most of cases, but 2 female cases from group A with multiple combined risk factors had needed to use low dose steroid eye drops and night eye anti-inflammatory ointments in addition to relatively longer period of lubricating eye drops using to reduce dry eye symptoms. According to the present study results, the most important risk factor for postoperative dry eye symptoms was excision of OOM strip during ULB. History of lubricating eye drops using was also associated with increased incidence of postoperative dry eye symptoms. Hypothyroidism and diabetes mellitus (DM) were suspected risk factors which have needed to be proven by further specific studies. Changes of TFBUT and reported dry eye symptoms following ULB were temporary and reversible in all cases of the present study.

Conclusion: Excision of OOM strip during ULB was associated with temporary statistically significant decrease in TFBUT and was also associated with more occurrences of reversible dry eye symptoms. Avoiding excision of OOM strip during ULB (if not indicated) was proven to minimize the occurrence of postoperative dry eye symptoms especially for patients with history of DES, for patient with repeated ULB, and those with systemic risk factors such as DM and thyroid dysfunctions.

Key words: upper lid blepharoplasty, orbicularis oculi muscle, dry eye, tear film break up time.

\section{INTRODUCTION}

Upper lid blepharoplasty (ULB) is one of the most common facial plastic surgeries performed, which can be done for functional or aesthetic indications (Hahn et al., 2016).

Functional indications of ULB include significant upper eyelid dermatochalasis encroaching on the visual axis and impeding peripheral vision, associated upper eyelid entropion or lashes ptosis causing ocular surface irritation, and severe blepharitis and excessive dermatochalasis affecting prosthetic function in the anophthalmic socket. Cosmetic indications of ULB include aesthetically displeasing dermatochalasis and upper eyelid fullness. The foundation of successful ULB begins with a complete preoperative evaluation, a discussion of expectations, the selection of the appropriate surgical technique, and a discussion of potential complications (Yang et al., 2017).

Potential complications of blepharoplasty include: Hemorrhage, infection, corneal abrasion, ptosis, diplopia, eyelids numbness, dry eye syndrome (DES), lagophthalmos, lacrimal gland injury, residual excess skin, asymmetric eyelid crease, noticeable surgical scarring and sulcus deformity (Sniegowski et al., 2014).

It is actually common to experience dry eye in the early healing period after eyelid surgery. There are number of reasons for this but the bottom line is that it is usually self-limited and improves over a short period of time when the eyelid edema subsides. While rarely possible, lasting dry eyes can occur. This may be due to inflammatory changes to the lacrimal gland The precipitation of DES can frequently induce patient anxiety even if the aesthetic result is satisfactory (Whipple et al., 2013).

Overzealous excision of soft tissue (mostly skin and orbicularis oculi muscle (OOM)), or denervation (OOM dysfunction caused by muscle manipulation) can result in poor eyelid function that is typically transient. Prolonged denervation, however, can result in poor lid position which may be permanent. OOM denervation causes defective eye blinking and without a brisk 
natural blink, tears are not properly spread over the surface of the eye leading to tear film changes and long term DES (Briceno et al., 2015).

ULB can be performed in most patients with dry eyes by excising a conservative amount of skin and, most important, by preserving all of the OOM and its innervation (Prischmann et al., 2013).

The exceptions to OOM preservation are in Asian patients undergoing ULB, in patients lacking a crease or having an asymmetrical crease, and in patients with severe dermatochalasis associated with excessive redundant OOM tissue (Park, 2015).

Over-excision of skin and muscle with the induction of severe exposure symptoms is less frequently seen after primary surgery and in younger people. On the other hand, increased symptoms are more prevalent in secondary surgery, especially in older people; premenopausal females; and also in patients with thyroid ophthalmopathy, treated or undiagnosed, in whom symptoms may be severe (Whipple et al., 2013).

The time required for the ocular surface to lose cohesive surface wetting after each blink is referred to tear film break up time (TFBUT). TFBUT test is a clinical test used to assess for evaporative DES. TFBUT is also abnormal in aqueous tear deficiency ( $\mathrm{Su}$ et al., 2014). To measure TFBUT, 2\% fluorescein or impregnated fluorescein strip moistened with non-preserved saline is instilled into the lower fornix. The patient is asked to blink several times and then not to blink while the tear film is observed under a broad beam of cobalt blue slit lamp illumination. The TFBUT is recorded as the number of seconds that elapse between the last blink and the appearance of the first dry spot in the tear film. A TFBUT under 10 seconds is considered abnormal (Peng et al., 2013).

ULB associated DES is underreported and probably not discussed often enough with patients prior to surgery and after surgery (Yang et al., 2017).

The present work aimed to study the changes of TFBUT and to compare incidence and severity of dry eye symptoms after ULB with and without excision of OOM strip.

\section{PATIENTS AND METHODS}

This interventional comparative study included 40 eyes of 20 patients (14 females and 6 males, mean (M) age was $57.05 \pm 4.80$ years old, range $(R)$ was 51 65) who had bilateral ULB. All cases were operated and followed up at AlHussein University Hospital and Oyoun Eye Center (Cairo) between January, 2017 and November, 2017. All patients provided preoperative informed consent. TFBUT was measured for each patient preoperatively, after one week and after one month postoperatively. Patients with continuous symptoms of dry eye postoperatively were followed up until three months. By retrospective analysis, patients were divided into two groups:

- Group A: Eight patients (5 females and 3 males, $M$ age was $58.38 \pm 5.53$ years old, R: 52-65) who had 16 ULB surgeries including OOM strip excision.

- Group B: Twelve patients (9 females and 3 males, $M$ age: $56.17 \pm 4.26$ years old, R: 51-62) who had 24 ULB surgeries with preservation of OOM. 
Preoperative evaluation: General history was taken including hypertension, diabetes mellitus (DM), thyroid dysfunctions, allergies and systemic medications. Patients with uncontrolled chronic systemic diseases were excluded, and patients on aspirin or non-steroidal anti-inflammatory medications were advised to stop them for suitable period preoperatively after specific medical consultation. Full ophthalmologic history was taken including DES, lids or ocular surgeries including corneal and refractive surgeries, and contact lenses use. Full ophthalmologic examination was performed including best corrected visual acuity (BCVA), slit lamp examination, dry eye assessment (including tear film, corneal examination, TFBUT and Schirmer's tests), and ocular motility. External eye and lid appearance were noticed, and lid crease height, palpebral fissure and marginal reflex distance were measured. Patients with exophthalmos, lid retraction, ptosis, brow ptosis, entropion or ectropion were excluded. All ULB surgeries in the present study were mainly for cosmetic indications. Preoperative and postoperative photographs were taken for documentation.

Operative details: Upper lid marking with surgical ink was performed with patient in an upright-seated position. The lower edge of the wound was marked at the normal lid crease taking into consideration the gender variations and its medial end was lateral to the punctum. Marking the upper edge of the wound was done aided by modified pinch technique (Bhattcharrjee et al., 2017) using 2 toothless forceps and according to Flowers' rule (Flowers and Flowers, 1993) to leave at least $20 \mathrm{~mm}$ of skin between the eyebrow and lid margin to excise the excess redundant skin without causing postoperative lagophthalmos.

Monitored care general sedation associated with upper lid subcutaneous slow local anesthesia injection (2-3 $\mathrm{ml}$ of $2 \%$ lidocaine with 1:100,000 epinephrine using a 27 gauge needle) were used in association with topical $0.4 \%$ benoxinate hydrochloride administration.

The skin flap was excised using traditional scalpel and scissors. 2-3 mm strip of OOM was only excised if there was excess redundant muscular tissue. In the other cases the OOM was preserved and small 2 button holes horizontal incisions centrally and medially through the OOM and then through the orbital septum were done to expose the underlying fat pads. The excess of large central yellow fat bad and the excess of smaller paler medial fat pad were then excised by clamping the bulging fat cautiously without pulling and then by using the bipolar cautery to prevent bleeding. Closure of the OOM incision was done by two suture centrally and one suture medially using 6/0 vicryl sutures passing through the edge of levator aponeurosis for crease fixation and the skin was finally closed using continuous subcuticular 6/0 prolene sutures. Postoperative ice bag application was done for 5 minutes and then topical antibiotic and steroid ointment with steristrips were used over the wound.

Postoperative care: Postoperative examination was performed early in the few postoperative hours and then regularly to exclude potential postoperative complications. All patients were advised to use cold packs every two 
or three hours for 15 minutes at a time during the 1 st postoperative 48 hours to reduce the usual postoperative lid swelling and bruising, and to use warm compresses after 48 hours to promote wound healing. Combined antibiotic and steroid eye ointment was used over the wound by each patient 4 times/ day for the first postoperative week and reduced gradually over the next two weeks. The nonabsorbable sutures were removed 5 days after surgery.

Follow up: Full ophthalmologic examination including cornea and tear film examination was done at each follow up visit. TFBUT test was done 2 days after removal of sutures (at the 7th postoperative day) and then after one month postoperatively. Symptoms and severity of dry eye were questioned postoperatively until the end of follow up period.

\section{Statistical analysis:}

The changes of TFBUT of participants were statistically analyzed using the paired t-test. The data obtained including the $\mathrm{M}$ and the SD were used to obtain $\mathrm{P}$ value, and $P$ value $\leq 0.05$ was considered statistically significant.

\section{RESULTS}

Three patients in group A and four patients in group B had history of controlled DM, the M duration of DM was 4.7 years (R: 2-8). Two female patients (one in each group) had history of controlled stable hypothyroidism. Four patients in group A and Six patients in group B had positive preoperative history of occasional use of lubricating eye drops for control of mild dry eye symptoms without preoperative complaints. No patients had history of ophthalmic surgeries such as refractive eye and cataract surgeries in the last 6 preoperative months. One female patient in group A had had previous ULB 3 years before. No patients had preoperative signs of severe dry eye such as superficial punctuate keratitis or filamentary keratitis, and no patients had history of recurrent corneal erosions. During the postoperative follow up period, none of the patients had any symptomatic corneal complications. None of them had postoperative lagophthalmos or required further surgical interference.
Patients were satisfied with the cosmetic outcomes.

There was a statistically significant decrease in TFBUT $M$ values in group A from $11 \pm 1.37$ s preoperatively to be 9.69 $\pm 2.18 \mathrm{~s}$ after one week postoperatively $(\mathrm{P}=0.0003)$. This decrease in TFBUT $\mathrm{M}$ value in group A was temporary and reversible as there was statistically significant increase of TFBUT $M$ value from 9.69 $\pm 2.18 \mathrm{~s}$ after one week postoperatively to be $10.56 \pm 1.86 \mathrm{~s}$ after one month postoperatively which was near to the preoperative value $(\mathrm{P}=0.0023)$.

In group $\mathrm{B}$, there were non-statistically significant changes in TFBUT $M$ values. There was non-statistically significant decrease in TFBUT $M$ values in group B from $11.42 \pm 1.86 \mathrm{~s}$ preoperatively to be $11.29 \pm 1.99 \mathrm{~s}$ after one week postoperatively $(\mathrm{P}=0.4259)$ and nonstatistically significant increase of TFBUT $\mathrm{M}$ values from $11.29 \pm 1.99 \mathrm{~s}$ after one 
week postoperatively to be $11.33 \pm 1.86$

after one month postoperatively (Table 1).

Table (1): Changes of TFBUT in groub A and B

\begin{tabular}{|c|c|c|}
\hline $\begin{array}{ll}\text { TFBUT (in seconds) } & \text { Groups } \\
\end{array}$ & Group A & Group B \\
\hline Preoperative TFBUT & $\begin{array}{l}\text { M: } 11 \pm 1.73 \\
\text { R: } 9-13\end{array}$ & $\begin{array}{l}\text { M: } 11.42 \pm 1.86 \\
\text { R: } 9-15\end{array}$ \\
\hline TFBUT one week postoperatively & $\begin{array}{l}\text { M: } 9.69 \pm 2.18 \\
\text { R: } 7-13\end{array}$ & $\begin{array}{l}\text { M: } 11.29 \pm 1.99 \\
\text { R: } 8-14\end{array}$ \\
\hline TFBUT one month postoperatively & $\begin{array}{l}\text { M: } 10.56 \pm 1.86 \\
\text { R: } 8-13\end{array}$ & $\begin{array}{l}\text { M: } 11.33 \pm 1.86 \\
\text { R: } 9-15\end{array}$ \\
\hline
\end{tabular}

Four patients (three females and one male) from group A (50\%) and 2 patients (one female and one male) from group B $(16.7 \%)$ had recorded symptoms of postoperative dry eye in the first follow up visit in the form of feeling of grittiness, redness, burning sensation and stringy discharge. These symptoms were more severe in patients of group A. The two patients who had recorded dry eye symptoms from group B had history of occasional use of lubricating eye drops and one of them was diabetic male and the other one was female with history of stable controlled hypothyroidism. Frequent use of lubricating eye drops and gels for one week was enough to reduce the patients' discomfort. However, two patients from group A (52 years old

\section{DISCUSSION}

It is quite common to have some dry eye symptoms after ULB. The inflammation after surgery can lead to reduced tear production and dry eyes. This is temporary in the vast majority of cases. With excision of musculocutaneous flap, OOM does not tend to work well for at least few weeks. The tear film is thinner, and the blink reflex decreased leading to female patient with history of controlled DM and stable controlled hypothyroidism, and 55 years old female with history of controlled DM, hypertension and previous ULB 3 years before) have needed to use low dose steroid eye drops and night eye anti-inflammatory ointments for few days, in addition to the lubricating eye drops to reduce the dry eye symptoms. Those two patients had also past history of occasional use of lubricating eye drops preoperatively, and after the 1st postoperative month they recorded continuous mild dry eye symptoms which improved significantly before the $3 \mathrm{rd}$ postoperative month by regular use of lubricating eye drops only with lesser frequency.

DES. Only few and relatively old studies had concerned with incidence and risk factors of DES following blepharoplasty (Yang et al., 2017).

(Prischmann et al., 2013) have shown that almost 27 percent of patients reported dry eye after surgery, and another 26 percent had chemosis. Those side effects happened more often in people who have had both upper and lower eyelid lifts. 
More aggressive surgical techniques also appeared to increase the risk of dry eye. (Sniegowski et al., 2014) have recommended staging, by operating on the upper eyelids first, followed by the lower eyelids several weeks later, excising conservative amounts of skin, muscle, and fat.

The present study included patients who had only bilateral ULB surgeries. TFBUT was used as a tool for dry eye documentation. OOM strip excision in the present study was done only when indicated .

Reversible temporary dry eye symptoms appeared in 6 patients in the present study (30\%): 4 patients from group A (50\%), and 2 patients from group B (16.7\%), and the symptoms were relatively more severe in patients of group A. These results were supportive to (Prischmann et al., 2013) that more aggressive surgical techniques increase the risk of dry eye.

(Saadat and Dresner, 2004) had evaluated the safety of performing ULB without OOM excision in patients with DES. This study had included all patients with subjective preoperative DES and/or an abnormal basic Schirmer's test result. The study showed that $8 \%$ incidence of worsening of dry eyes after ULB, and the patients required increased amounts of artificial tears only to control the dry eye symptoms. They concluded that ULB can be safely performed in patients with preoperative DES if the OOM and its innervation were preserved. (Fagien, 2004) had reported that Schirmer's test has little value in screening or prevention of DES after blepharoplasty for those at high risk because it is a technique dependent and varying results are common in the same patient tested by different examiners .

In contrast to (Saadat and Dresner, 2004) study, the present study excluded patients with preoperative significant DES or abnormal Schirmer's tests results to evaluate the pure effect of OOM excision which may be indicated in ULB on occurrence or aggravation of postoperative dry eye symptoms. The present study used Schirmer's tests preoperatively to exclude patients with low tear production and used TFBUT test preoperatively and at regular postoperative follow up visits because TFBUT is practical, inexpensive, and easy to perform and specific for evaluation of evaporative DES related to defective blinking .

According to the present study results, the most important risk factor for postoperative dry eye symptoms was excision of OOM strip during ULB. Fifty percent of patients who had OOM strip excisions recorded postoperative dry eye symptoms .

History of lubricating eye drops using was also associated with increased incidence of postoperative dry eye symptoms in the present study. Four patients (2 from each group) had history of occasional use of lubricating eye drops and reported postoperative dry eye symptoms. According to the present study results, the two patients with history of hypothyroidism and 3 out of 7 patients with history of DM complained of postoperative dry eye symptoms. So hypothyroidism and DM were suspected risk factors. This needed to be proven by further specific studies. 
(Yang et al., 2017) had reported that patients who had prior blepharoplasty, patients who had overzealous ULB associated with musculocutaneous flap excision or brow lift were susceptible to dry eye symptoms following ULB. Patients with significant brow ptosis in need for brow lift were excluded from the present study.

\section{CONCLUSION}

Excision of OOM strip during ULB was associated with temporary statistically significant decrease in TFBUT and more occurrence of reversible dry eye symptoms. History of dry eye symptoms, preoperative careful corneal and tear film examination and preoperative discussion with the patient about the potential risk of DES following ULB, especially those with risk factors, were proved to be important during the preoperative evaluation for ULB. If OOM strip excision was not indicated, preserving OOM and its innervation was concluded to reduce the risk of postoperative DES. Routine use of lubricating eye drops for few weeks following ULB was concluded to reduce the postoperative discomfort especially for patients with history of DES and those with systemic risk factors such as DM and thyroid dysfunctions.

\section{REFERENCES}

1. Bhattcharrjee $K$, Misra DK and Deori $\mathbf{N}$ (2017): Updates on upper eyelid blepharoplasty, Indian J Ophthalmol., 65 (7): 551-558.

2. Briceno CA, Zhang-Nunes SX and Massry GG (2015): Minimally invasive surgical adjuncts to upper blepharoplasty, Facial Plast Surg Clin N Am., 23 (1): 137-151.
3. Fagien S (2004): Reducing the incidence of dry eye symptoms after blepharoplasty, Aesthetic Surg J., 42 (5): 464-468.

4. Flowers RS and Flowers SS (1993): Precision planning in blepharoplasty: the importance of preoperative mapping, Clin Plast Surg., 20 (1): 303.

5. Hahn S, John B. Holds and Steven M (2016): Upper lid blepharoplasty, Facial Plast Surg Clin N Am., 24 (1): 119-127.

6. Park DD (2015): Aging Asian upper blepharoplasty and brow. Semin Plast Surg., 29 (3): 188-200.

7. Peng CC, Tan B, Lin M and Radke C (2013): Black spot formation and fluorescence tear break up time. Investigative Ophthalmology \& visual science, 54 (15): 943.

8. Prischmann T, Sufyan A, Ting JY, Ruffin C and Perkins SW (2013): Dry eye symptoms and chemosis following blepharoplasty: a 10 year retrospective review of 892 cases in a single surgeon series, JAMA Facial Plast Surg., 15 (1): 39-46.

9. Saadat D and Dresner SC (2004): Safety of blepharoplasty in patients with preoperative dry eyes, Arch Facila Plast Surg., 6 (2): 101-104.

10. Sniegowski B, Davies, $B$ Hink $E$ and Vikram D (2014): Complications following blepharoplasty, Expert Review of Ophthalmology, 9 (1): 341-349.

11. Su TY, Chang SW, Yang CJ and Chiang HK (2014): Direct observation and validation of fluorescent tear film break up patterns by using a dual thermal fluorescent imaging system, Biomed Opt Express, 5 (8): 2614-2619.

12. Whipple KM, Lim LH and Korn BS (2013): Blepharoplasty complications: prevention and management, Clin Plast Surg., 40(1): 213-224.

13. Yang P, Audrey C Ko, Don O Kikkawa and Bobby S Korn (2017): Upper Eyelid Blepharoplasty: Evaluation, Treatment, and Complication Minimization, Semin Plast Surg., 31 (1): 51-57. 


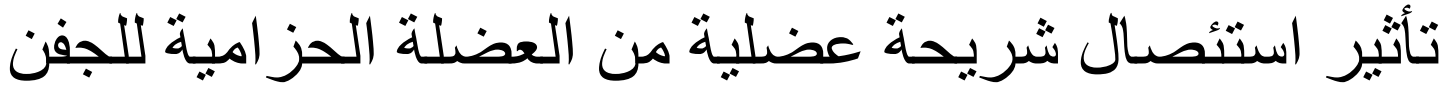

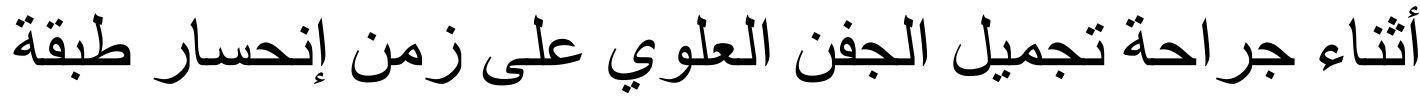

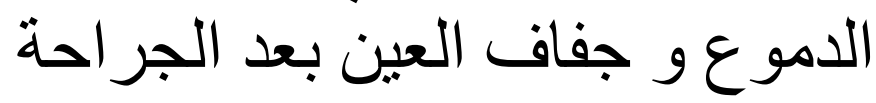
مصطفى فريد محمد النكلاوي

قسم طب و جراحة العين- كلية الطب (بنين)- جامعة الأزهر

خلفية البحث : إن حدوث أعر اض جفاف العين بعد جراحة تجميل الجفن العلوي أمرّ شَائع وإن كانت هذه

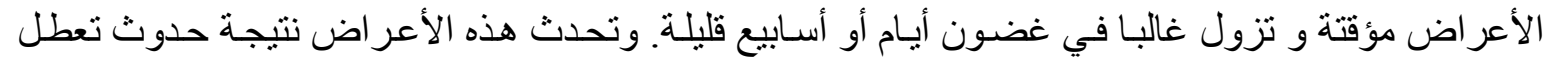

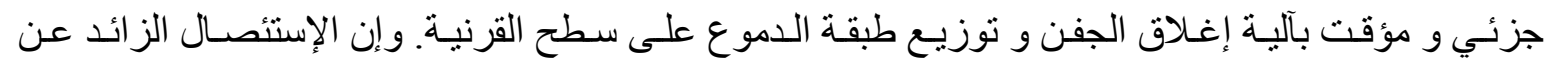

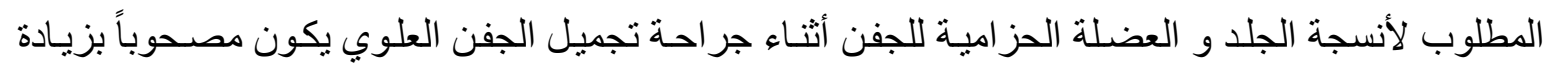
خطر حدوث جفاف العين بعد الجراحة.

الهذف من البحث: دراسـة تأثير إستئصسال شريحة عضلية من العضلة الحز امية للجفن أثناء جراحة تجميل

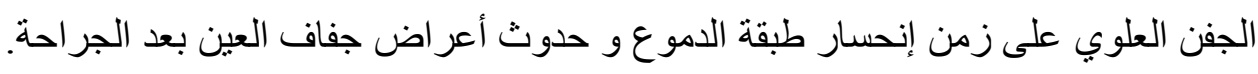

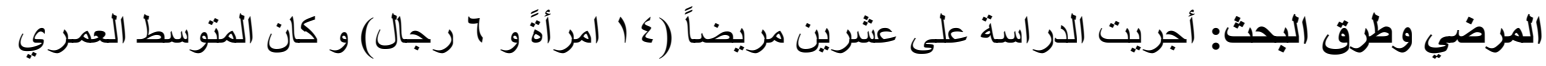

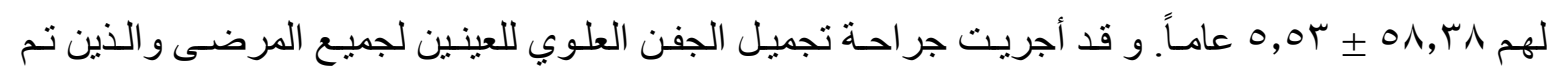

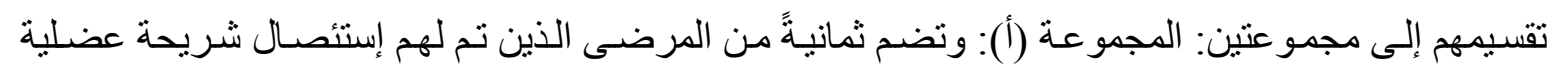

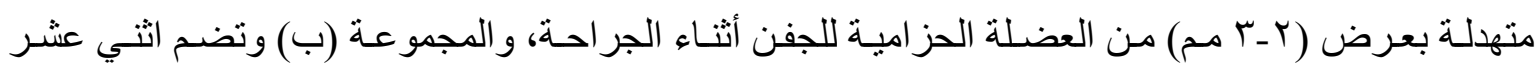

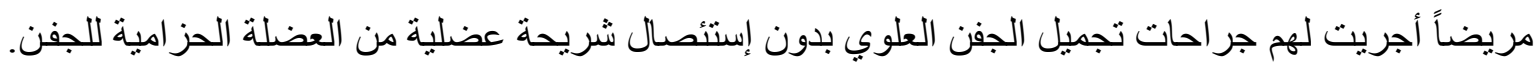

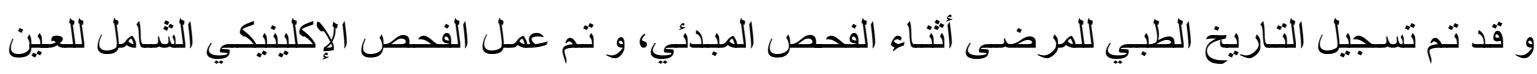

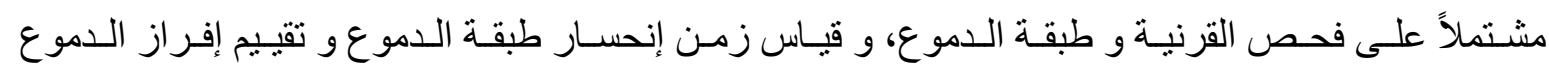

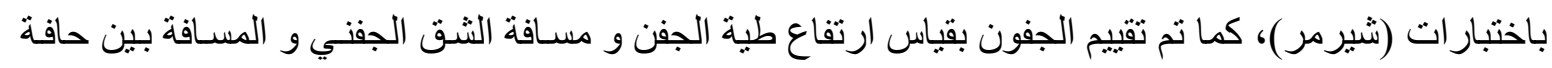

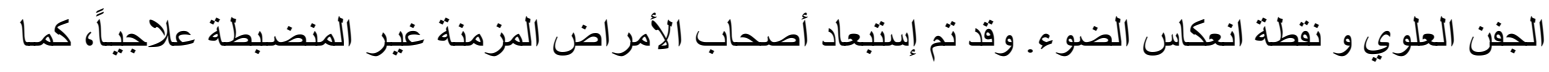

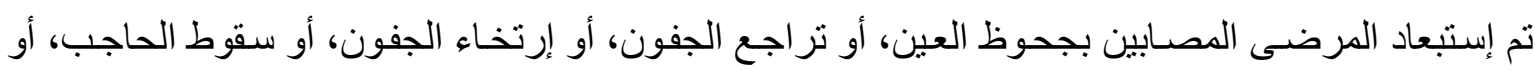

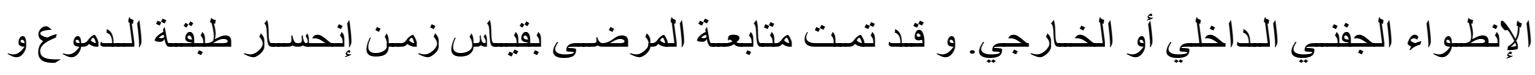

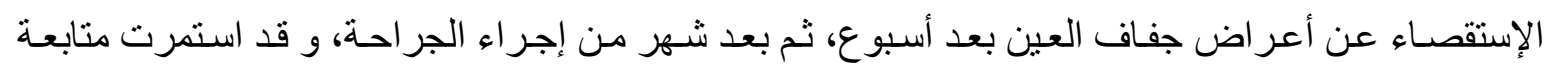

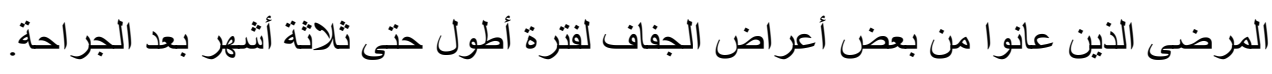

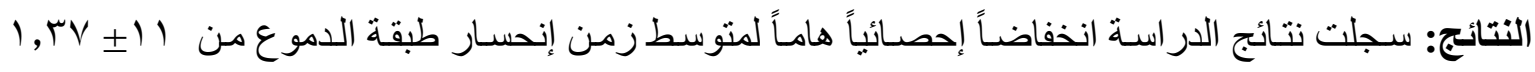

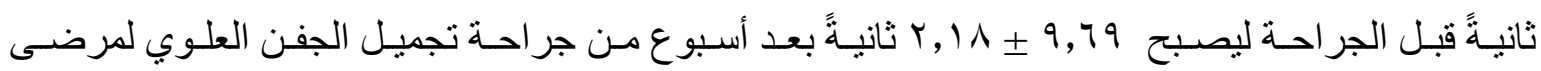




\section{MOSTAFA F MOHAMMED}

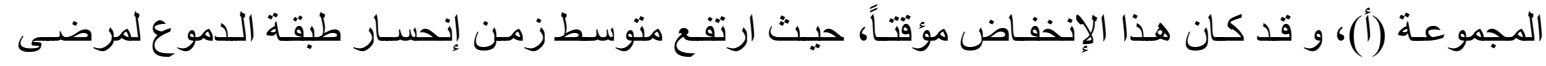

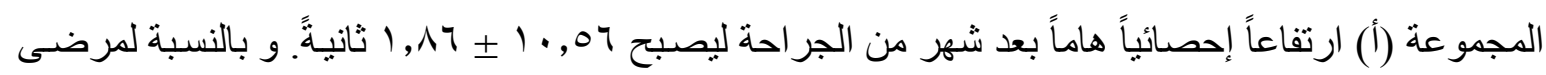

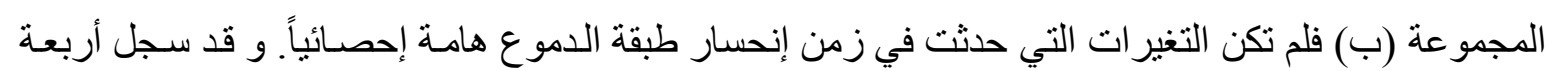

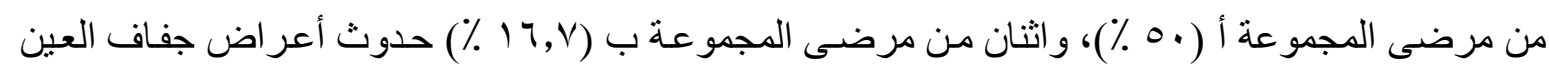

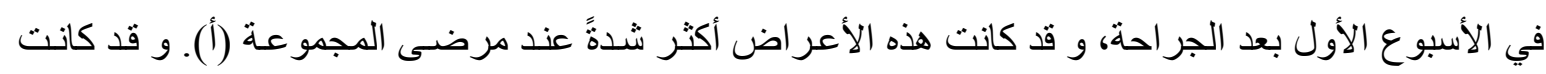

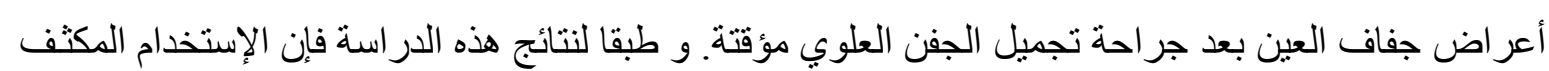

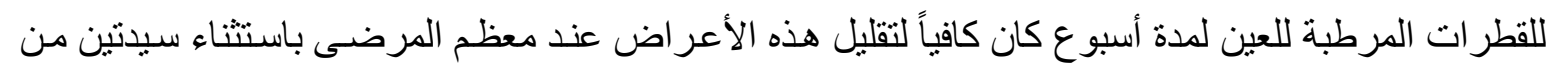

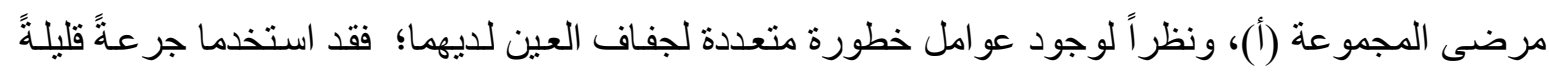

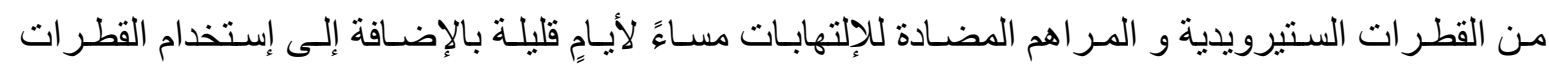

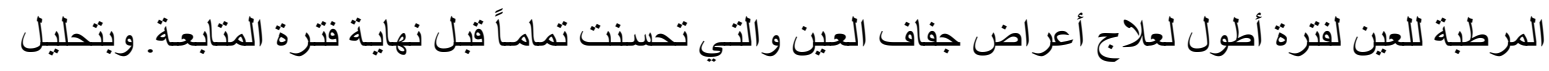

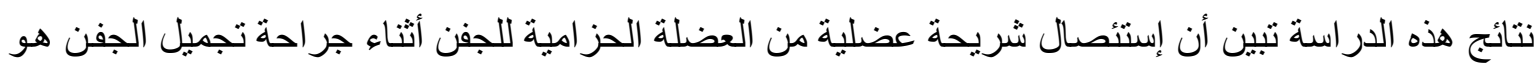

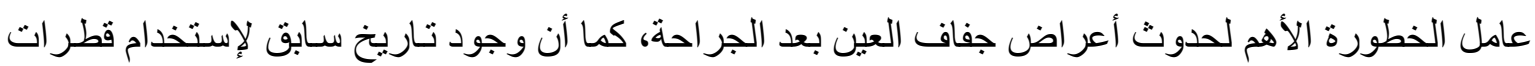

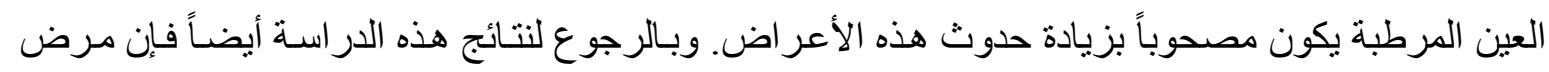

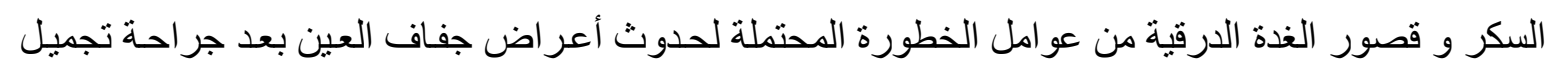

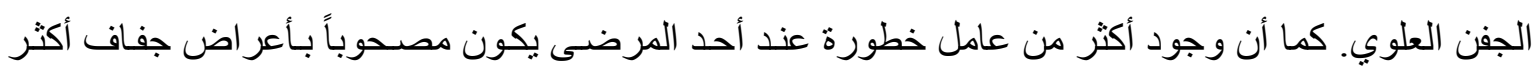

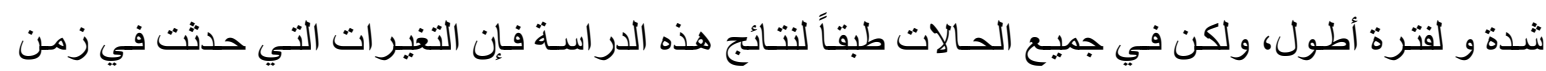
إنحسار طبقة الدموع وأعر اض جفاف العين بعد جراحة تجميل الجفن العلوي كانت جميعاً مؤقتَّ.

الاستتناج: إستئصال شريحة عضلية من العضلة الحزامية للجفن كان مصحوباً بانخفاض مؤقت لزمن إنحسار

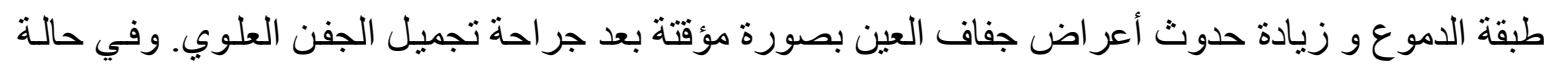

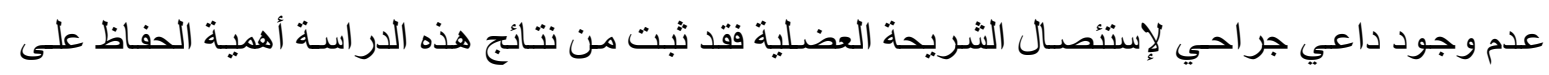

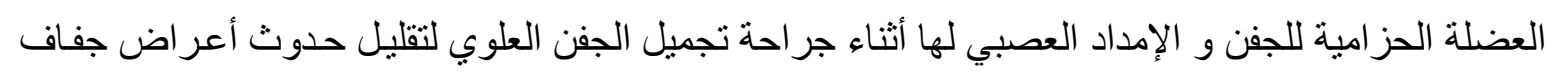

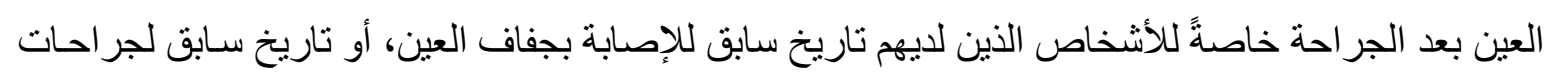

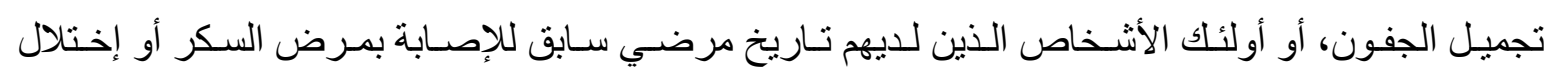

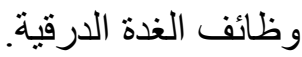

